\title{
Qualidade fisiológica de sementes Avena strigosa SCHREB. cv. comum submetidas ao envelhecimento acelerado
}

\author{
Physiological quality of Avena strigosa Schreb seed. cv. Common subjected to accelerated aging \\ Marciele Barbieri, Viviane Schons de Ávila, Caciara Gonzatto M, Gisele Noal, \\ Marlove Fátima Brião Muniz, Andrea Cristina Dörr \\ Universidade Federal de Santa Maria - UFSM
}

\section{Resumo}

Aveia preta vem sendo umas das principais espécies utilizadas como cobertura no inverno, tendo seu cultivo evoluído nos últimos anos no sul do Brasil. O trabalho objetivou avaliar o efeito dos tratamentos de sementes de aveia preta submetidas ao envelhecimento acelerado, em relação à qualidade fisiológica. Os tratamentos foram: Testemunha sem tratamento; fungicida químico Vincit ${ }^{\circledR} 50 \mathrm{SC}$; Vincit ${ }^{\circledR} 50 \mathrm{SC}+$ produto biológico Trichode ${ }^{\circledR} \mathrm{e}$ Trichodel $^{\circledR}$. As doses foram seguidas conforme a indicação anexada a cada um desses produtos para a cultura da aveia. As sementes, após tratadas, foram submetidas ao envelhecimento acelerado por $0,24,48$ e 72 horas a $41^{\circ} \mathrm{C}$. As variáveis analisadas foram: germinação, primeira contagem, plântulas anormais, sementes mortas, comprimento de parte aérea e comprimento radicular. Os resultados mostram que as sementes tratadas com o fungicida químico associado ao produto biológico, e quando este foi utilizado isoladamente, apresentaram o melhor desempenho, tanto para a germinação como para o desenvolvimento das plântulas submetidas, quando submetidas a 48 horas de estresse por envelhecimento acelerado. O período de envelhecimento de 72 horas prejudicou a qualidade fisiológica das sementes, independente do tratamento utilizado. Conclui-se que as sementes tratadas com o produto Trichodel ${ }^{\circledR}$ apresentaram um comportamento distinto dos demais, porém, com uma queda numérica menos drástica.

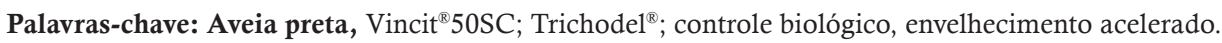

\begin{abstract}
Black oats has been one of the main species used as cover in winter, having its cultivation evolved in recent years in southern Brazil. The work aimed to evaluate the effect of the treatments of black Oat seeds subjected to accelerated aging. The treatments were: Witness without

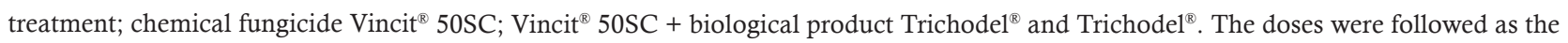
indication attached to each of these products for the cultivation of oats. The seeds, after treated were submitted to accelerated aging by 0,24 , 48 and 72 hours to $41^{\circ} \mathrm{C}$. The variables analyzed were: germination, first count, abnormal seedlings, seeds, length of shoot and root length. The results show that the seeds treated with fungicide chemical associated with the organic product, and when it was used alone, showed the best performances for both germination and development of seedlings subjected to 48 hours of stress by accelerated aging. The aging period of 72 hours has damaged the physiological quality of seeds, regardless of the treatment used. It is concluded that seeds treated with the product Trichode ${ }^{\circledR}$ presented a distinct behavior from others, however, with a numerical drop less drastic.

keywords: black Oats, Vincit ${ }^{\circledR} 50 S C$; Trichodel ${ }^{\circledR}$; biological control, accelerated aging.
\end{abstract}




\section{INTRODUÇÃO}

A aveia consiste em uma das principais culturas utilizadas no Sul do Brasil, principalmente porque é destinada para a alimentação animal na forma de forragem, silagem, além de ser utilizada na forma de grãos para a diversificação na exploração agrícola. A aveia preta apresenta altas produções de massa verde, e é menos vulnerável às moléstias resistindo melhor ao pisoteio, uma vez que seus grãos não apresentam valor industrial. De acordo com os dados da Comissão Brasileira de Pesquisa de Aveia - CBPA (2006), o maior consumo de aveia pelos animais é a partir do feno e da silagem, já os seus grãos destinam-se para a fabricação de ração animal. No entanto, cerca de $20 \%$ da produção de aveia, mundialmente, é servida como alimento aos seres humanos. Além disso, Souza et al. (2009) relata que esta gramínea é adaptada tanto a região temperada quanto subtropical e tem destaque na produção de massa seca, abundante massa radicular (o que contribui para a melhor aeração e descompactação do solo), melhorando as condições físicas e biológicas do solo além de controlar doenças e plantas invasoras.

$\mathrm{O}$ vigor das sementes afeta diretamente a emergência das plântulas e o estabelecimento das culturas no campo, podendo assim gerar perdas na produção. Estes aspectos de desempenho incluem a taxa e uniformidade de germinação de sementes e crescimento de plântulas em laboratório (SCHUCH et al., 1999), emergência e crescimento de plântulas no campo (SCHUCH e LIN, 1982; SCHUCH, 1999), habilidade de emergência de plântulas em condições ambientais desfavoráveis, além de afetar o crescimento e rendimento da planta.

De acordo com Balardin e Lock (1987), o tratamento de sementes consiste em uma alternativa concisa e econômica, que garante maiores percentuais de germinação e emergência das plântulas, isto é, resulta no retardamento da infecção primária das sementes e, reduz o inóculo de patógenos radiculares e de solo. Além dos fungicidas químicos, destacam-se os produtos bioprotetores que surgiram no intuito de reduzir a utilização de pesticidas sintéticos, os riscos aos operadores e os possíveis prejuízos ao meio ambiente.

Os testes utilizados na definição da qualidade fisiológica das sementes consistem na germinação e vigor, dentre estes se destaca o teste de envelhecimento acelerado, o qual foi desenvolvido com o propósito de avaliar o vigor de sementes, com o intuito de estimar o potencial de armazenamento das mesmas principalmente em relação à umidade e temperaturas elevadas. De acordo com Marcos Filho et al. (1987), neste teste, ao aumentar a temperatura e a umidade, a taxa de deterioração das sementes também irá elevar-se, sendo a deterioração variável de acordo com o seu vigor inicial.

Diante do exposto, o objetivo do trabalho consiste em estudar a eficiência da cultura da aveia preta cv. comum a partir do efeito dos tratamentos de sementes desta cultivar submetidas ao envelhecimento acelerado.

\section{METODOLOGIA}

\section{I Aquisição das sementes e recepção no laboratório}

As sementes de aveia preta cv. comum foram produzidas no município de Júlio de Castilhos-RS, e obtidas através de um produtor local. Os trabalhos foram desenvolvidos no Laboratório de Fitopatologia Elocy Minussi do Departamento de Defesa Fitossanitária da Universidade Federal de Santa Maria, durante o período de março a outubro de 2012, e em área experimental neste mesmo município, também neste mesmo intervalo de tempo, e em área experimental neste mesmo município. As sementes de aveia preta foram submetidas a diferentes tratamentos e ao envelhecimento acelerado.

\subsection{Submissão das sementes ao tratamento químico e biológico.}

$\mathrm{O}$ tratamento químico utilizado para o controle com fungicida consistiu no Vincit ${ }^{\circledR}$ 50SC (grupo químico triazol sistêmico, suspensão concentrada (SC) e indicado para uso em tratamento de sementes nas culturas de algodão, aveia, cevada, feijão, soja e trigo), o qual é registrado no Ministério da Agricultura, Pecuária e Abastecimento - MAPA. Para a cultura da aveia este produto controla Helmintosporiose (Drechslera avenae) e Mancha-de-Alternaria (Alternaria alternata).

O tratamento biológico empregado foi produto Trichodel ${ }^{\circledR,}$ o qual é um produto biológico composto de linhagens selecionadas do fungo Trichoderma spp. e que inibe o desenvolvimento de fungos patogênicos presentes no solo e sobre as plantas. 
As sementes foram submetidas aos tratamentos: T1 (testemunha), T2 (Vincit ${ }^{\circledR}$ 50SC), T3 $\left(\right.$ Vincit $^{\circledR} 50 S C+$ Trichodel $\left.^{\circledR}\right)$ e T4 $\left(\right.$ Trichodel $\left.^{\circledR}\right)$ e as doses de cada produto foram seguidas conforme a indicação anexada a cada um desses produtos. Utilizou-se, para o produto Vincit ${ }^{\circledR} 50 \mathrm{SC}$, o recomendado $150 \mathrm{~mL}$ para $100 \mathrm{~kg}$ de aveia; então, 1,25 $\mathrm{kg}$ (quantidade utilizada para todo o experimento tanto a campo como em laboratório) de aveia resultando em 1,9 mL de Vincit ${ }^{\circledR} 50$ SC. Da mesma maneira, conduziu-se o tratamento das sementes com o produto biológico, ou seja, o recomendado para o produto Trichodel ${ }^{\circledR}$ é de $250 \mathrm{~mL} / \mathrm{saco}$ de $40 \mathrm{~kg}$ de aveia; então, 1,25 kg de aveia (quantidade utilizada para todo o experimento tanto a campo como em laboratório) resultando em $6,25 \mathrm{ml}$ de Trichodel $^{\circledR}$.

\subsection{Submissão das sementes ao estresse pelo envelhecimento acelerado}

Após o tratamento químico e biológico, as sementes de aveia preta foram expostas a uma temperatura que oscilou entre 40 e $43^{\circ} \mathrm{C}$, em diferentes tempos de exposição: 0 - $24-48$ e 72 horas. $\mathrm{O}$ teste de sanidade foi realizado utilizando oito repetições de 50 sementes. Estas foram colocadas em caixas acrílicas transparentes (gerbox) com dimensões de $11 \times 11 \times 3 \mathrm{~cm}$, previamente desinfetadas com solução de hipoclorito de sódio (1\%) e álcool (70\%).

Utilizaram-se como substrato duas folhas de papel-filtro, umedecidas com água destilada esterilizada. Adicionou-se $40 \mathrm{ml}$ de água, as sementes foram dispostas em uma tela do formato da gerbox, posicionadas na parte superior de forma que não entre em contato com água. A verificação da ocorrência de fungos foi feita com auxílio de microscópio estereoscópico, no qual as sementes foram observadas individualmente. A identificação dos fungos em nível de gênero foi realizada com microscópio óptico conforme explicam Barnett e Hunter (1999).

\subsection{Análise estatística}

As variáveis primeira contagem, germinação, plântulas anormais, sementes mortas, comprimento de parte aérea e comprimento de raiz; avaliadas a partir de sementes conduzidas ao envelhecimento acelerado, os tratamentos foram organizados em arranjo bi-fatorial em 4 tratamentos por 4 tempos, através da análise de regressão pelo programa estatístico.

\section{RESULTADOS E DISCUSSÃO}

Os resultados do teste de germinação para a Testemunha, Vincit ${ }^{\circledR} 50 \mathrm{SC}$ e Vincit ${ }^{\circledR} 50 \mathrm{SC}+$ Trichodel $^{\circledR}$, seguem uma tendência quadrática (Figura 1). Este resultado contraria aquele demonstrado por Marcos Filho \& Shioga (1981), o qual revela que o tratamento das sementes com fungicida não promoveu modificações acentuadas nas informações sobre o potencial fisiológico dos lotes avaliados, em estudos realizados com sementes de soja. Segundo Souza et al. (2009), o tratamento das sementes de aveia preta com fungicida possibilitou a utilização de períodos de condicionamento mais prolongados para a classificação dos lotes sem levá-los à ausência da germinação, quando comparado com o procedimento tradicional sem tratamento fungicida. Dessa forma, contradiz o resultado encontrado neste trabalho no que se refere às sementes tratadas com produto químico.

De acordo com a Figura 1, a germinação das sementes de aveia preta tratadas com o produto Vincit ${ }^{\circledR} 50 S C$ isolado, Vincit ${ }^{\circledR} 50 S C+$ Trichodel $^{\circledR}$ e para a Testemunha, é decrescente até o tempo de 72 horas, enquanto que para o Trichodel ${ }^{\circledR}$ as sementes se mostram mais vigorosas em relação aos demais tratamentos. Conseqüentemente, pode-se afimar que o uso de produto biológico no tratamento de sementes também beneficiou o potencial germinativo. Logo, o tratamento com Trichodel ${ }^{\mathbb{Q}}$ favoreceu a germinação das sementes mesmo em temperaturas elevadas.

De acordo com Santos (2006), os mecanismos envolvidos no aumento do crescimento e do rendimento, induzidos por espécies de Trichoderma spp., aparentemente, são o controle de patógenos secundários, os quais diminuem o crescimento, a atividade das raízes e a produção de fatores estimulantes de crescimento. Segundo Dias e Barros (1995) o teste de envelhecimento acelerado tem por objetivo acelerar a deterioração relacionada às condições ambientais adversas como alta umidade e temperatura, admitindo que sementes de alto vigor mantenham a qualidade em níveis aceitáveis. Dessa forma, classifica-se como sendo um teste de estresse. 


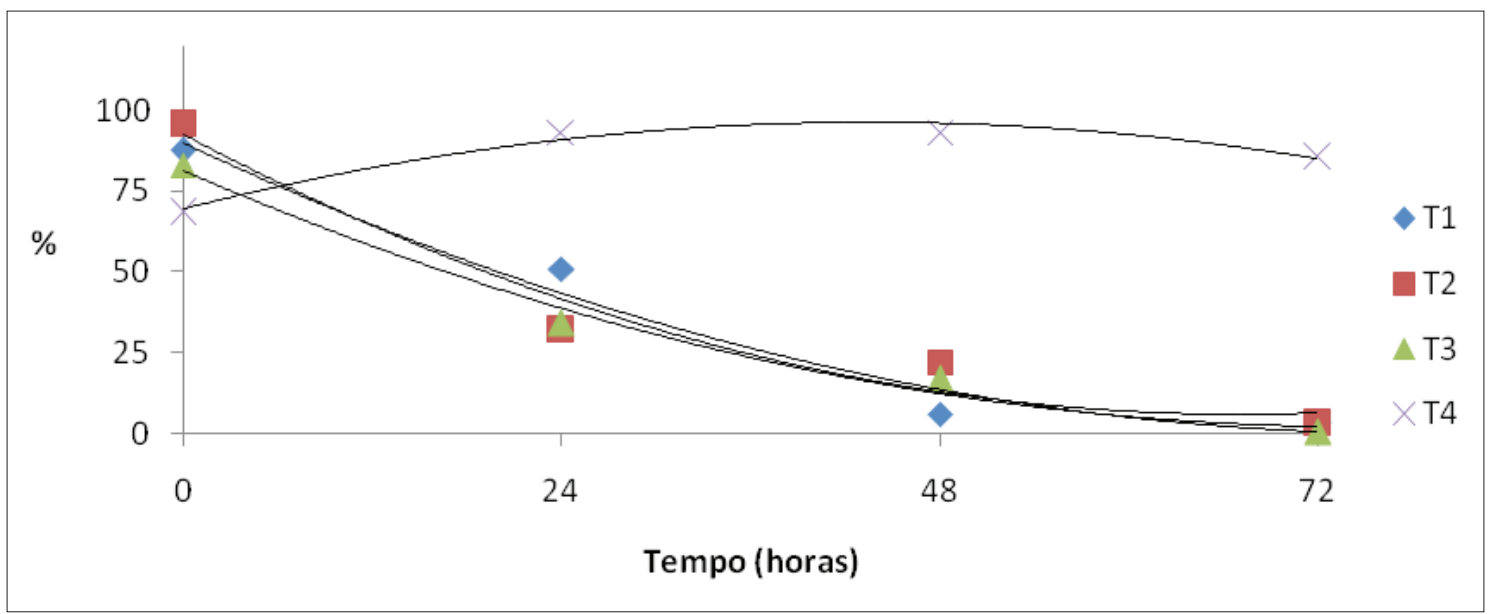

Figura 1 - Germinação (\%) de sementes de aveia preta cv. Comum submetidas a diferentes tratamentos e à diferentes tempos de envelhecimento acelerado. T1 - Testemunha; B - T2 - Vincit ${ }^{5} 5$ SC; T3 - Vincit ${ }^{5} 50 S C$ + Trichodel ${ }^{\circ}$ e T4 - Trichodel ${ }^{2}$. Santa Maria, 2012.

Fonte: Dados da pesquisa. BARBIERI, M. et. al (2012).

Tabela 1 - Equações das análises de regressão da variável germinação (\%) de Avena strigosa cv. Comum submetidas ao envelhecimento acelerado sob diferentes tratamentos.

\begin{tabular}{|c|c|c|}
\hline \multicolumn{2}{|c|}{ Germinação (\%) } \\
\hline Tratamento & Equação das análises de regressão & Nível de significância \\
\hline Testemunha - T1 & $\mathrm{y}=0,014 \mathrm{x}^{2}-2,290 \mathrm{x}+90,02$ & $\mathrm{R}^{2}=0,973$ \\
\hline Vincit $^{\circledR} 50 \mathrm{SC}-\mathrm{T} 2$ & $\mathrm{y}=0,019 \mathrm{x}^{2}-2,588 \mathrm{x}+92,37$ & $\mathrm{R}^{2}=0,959$ \\
\hline Vincit $^{\circledR} 50 \mathrm{SC}+$ Trichodel $^{\circledR}-\mathrm{T} 3$ & $\mathrm{y}=0,014 \mathrm{x}^{2}-2,117 \mathrm{x}+81,42$ & $\mathrm{R}^{2}=0,987$ \\
\hline Trichodel $^{\circledR}-\mathrm{T} 4$ & $\mathrm{y}=-0,014 \mathrm{x}^{2}+1,230 \mathrm{x}+69,27$ & $\mathrm{R}^{2}=0,962$ \\
\hline
\end{tabular}

Fonte: Dados da pesquisa. BARBIERI, M. et. al (2012).

Para a Testemunha (sem tratamento), as sementes tratadas com Vincit ${ }^{\mathbb{}} 50 \mathrm{SC}$ e para aquelas tratadas com Vincit ${ }^{\circledR} 50 \mathrm{SC}+$ Trichodel $^{\mathbb{R}}$, os resultados não foram expressivos em relação às plântulas anormais e sementes mortas (Figuras 2 e 3 , respectivamente). Além disso, indica que as plântulas anormais aumentam significativamente à medida que aumentou o tempo da exposição das sementes a elevadas temperaturas. Ao avaliar a variável sementes mortas, notou-se que é crescente até o período de, aproximadamente, 30 horas, posteriormente a este tempo a mortalidade diminuiu. No tratamento com Trichode ${ }^{\circledR}$ houve diferença para os demais tratamentos. De acordo com Santos (2006), os mecanismos envolvidos no aumento do crescimento e do rendimento, induzidos por espécies de Trichoderma spp., aparentemente são o controle de patógenos secundários, que diminuem o crescimento, a atividade das raízes e a produção de fatores estimulantes de crescimento.

Visto que nos demais tratamentos poucas sementes emergiram, devido ao tipo de tratamento, juntamente com a alta temperatura e muito tempo (horas) em contato com elevada umidade e calor, fizeram com que as sementes morressem mais e resultasse na percentagem maior de plântulas anormais. Dessa forma, o tratamento com Trichodel ${ }^{\circledR}$ apresentou percentagem relativamente baixa com relação à anormalidade e mortalidade das plântulas. Isso porque houve significativa germinação inicial das sementes, além do Trichoderma spp. contribuir para que houvesse um menor percentual de plântulas anormais e sementes mortas. 


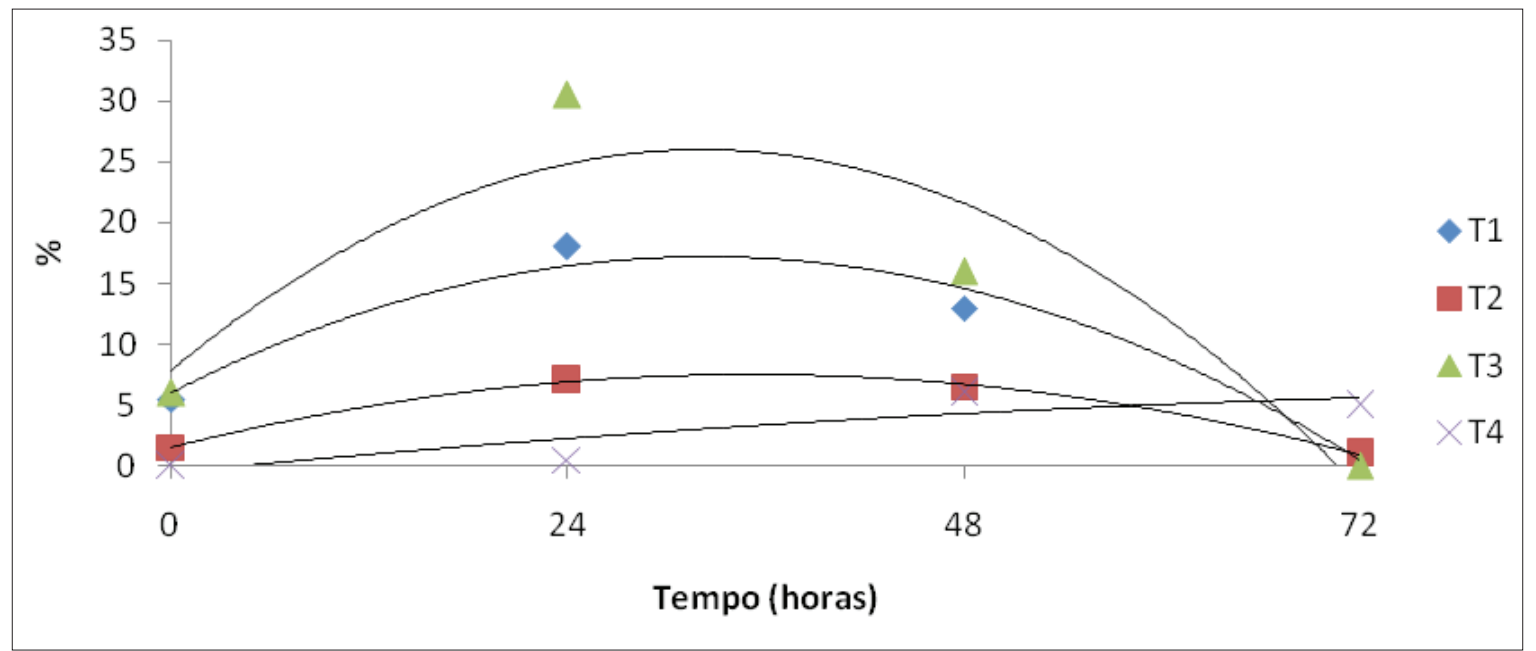

Figura 2 - Plântulas anormais (\%) de aveiapreta cv. Comum após diferentes tempos de envelhecimento acelerado. T1 - Testemunha; T2 - Tratamento químico (Vincit $\left.{ }^{\circledR} 50 S C\right) ;$ T3 - Químico + Trichoderma $\left(\right.$ Vincit $^{\circledR} 50 S C+$ Trichodel $\left.^{\circledR}\right)$ e T4 - Trichoderma $\left(\right.$ Trichodel $\left.^{\circledR}\right)$. Santa Maria, 2012.

Fonte: Dados da pesquisa. BARBIERI, M. et. al (2012).

Tabela 2 - Equações das análises de regressão da variável plântulas anormais (\%) de Avena strigosa cv. comum submetidas ao envelhecimento acelerado sob diferentes tratamentos.

\begin{tabular}{|c|c|c|}
\hline \multicolumn{3}{|c|}{ Plântulas anormais (\%) } \\
\hline Tratamento & Equação das análises de regressão & Nível de significância \\
\hline Testemunha - T1 & $\mathrm{y}=-0,010 \mathrm{x}^{2}+0,688 \mathrm{x}+6,025$ & $\mathrm{R}^{2}=0,968$ \\
\hline Vincit $^{\circledR} 50 \mathrm{SC}-\mathrm{T} 2$ & $\mathrm{y}=-0,004 \mathrm{x}^{2}+0,335 \mathrm{x}+1,55$ & $\mathrm{R}^{2}=0,998$ \\
\hline Vincit $^{\circledR} 50 \mathrm{SC}+$ Trichodel $^{\circledR}-\mathrm{T} 3$ & $\mathrm{y}=-0,017 \mathrm{x}^{2}+1,130 \mathrm{x}+7,875$ & $\mathrm{R}^{2}=0,868$ \\
\hline Trichodel $^{\circledR}-\mathrm{T} 4$ & $\mathrm{y}=-0,000 \mathrm{x}^{2}+0,132 \mathrm{x}-0,575$ & $\mathrm{R}^{2}=0,765$ \\
\hline
\end{tabular}

Fonte: Dados da pesquisa. BARBIERI, M. et. al (2012).

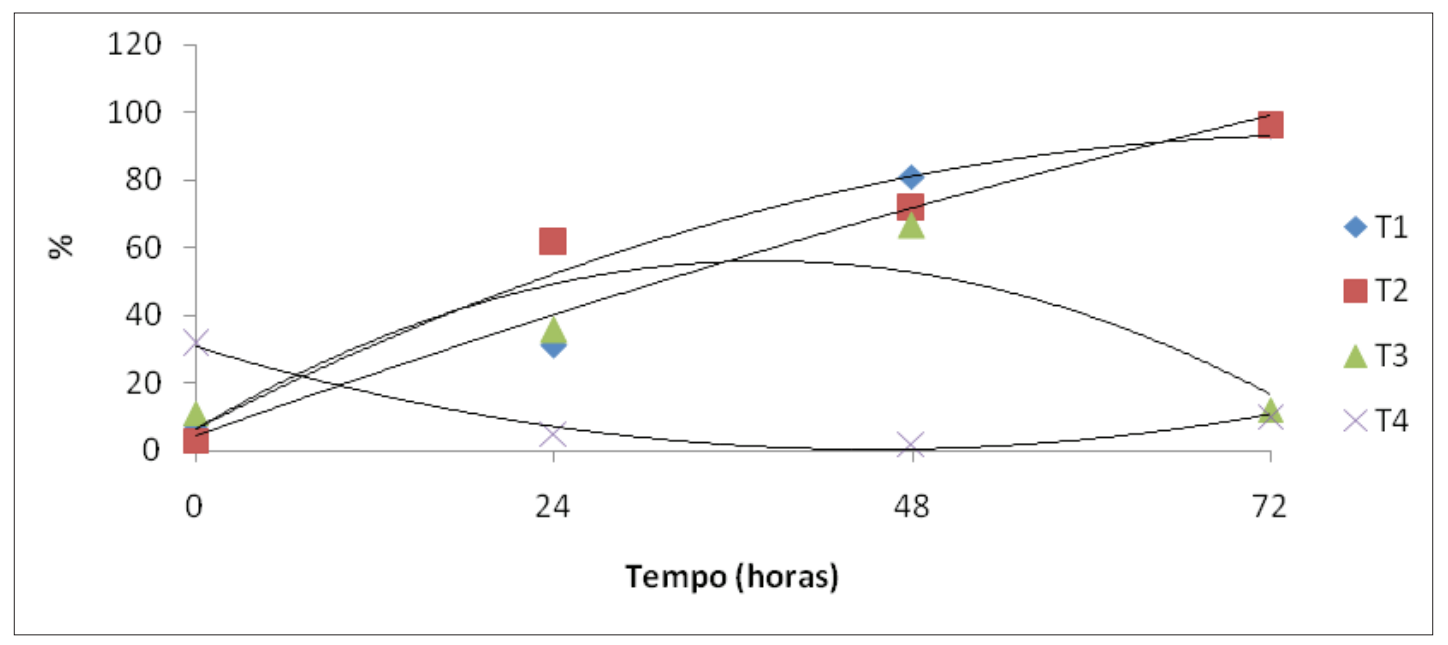

Figura 3 - Sementes Mortas (\%) de aveia preta cv. Comum após diferentes tempos de envelhecimento acelerado. T1 - Testemunha;

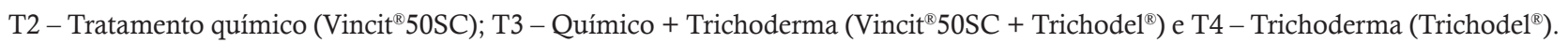
Santa Maria, 2012.

Fonte: Dados da pesquisa. BARBIERI, M. et. al (2012). 
Tabela 3 - Equações das análises de regressão da variável sementes mortas (\%) de Avena strigosa cv. comum submetidas ao envelhecimento acelerado sob diferentes tratamentos.

\begin{tabular}{|c|c|c|}
\hline \multicolumn{3}{|c|}{ Sementes mortas (\%) } \\
\hline Tratamento & Equação das análises de regressão & Nível de significância \\
\hline Testemunha - T1 & $\mathrm{y}=-0,003 \mathrm{x}^{2}+1,580 \mathrm{x}+4,425$ & $\mathrm{R}^{2}=0,963$ \\
\hline Vincit $^{\circledR} 50 \mathrm{SC}-\mathrm{T} 2$ & $\mathrm{y}=-0,014 \mathrm{x}^{2}+2,266 \mathrm{x}+6,15$ & $\mathrm{R}^{2}=0,957$ \\
\hline Vincit $^{\circledR} 50 \mathrm{SC}+$ Trichodel $^{\circledR}-\mathrm{T} 3$ & $\mathrm{y}=-0,034 \mathrm{x}^{2}+2,610 \mathrm{x}+6,4$ & $\mathrm{R}^{2}=0,792$ \\
\hline Trichodel $^{\circledR}-\mathrm{T} 4$ & $\mathrm{y}=0,015 \mathrm{x}^{2}-1,359 \mathrm{x}+30,87$ & $\mathrm{R}^{2}=0,985$ \\
\hline
\end{tabular}

Fonte: Dados da pesquisa. BARBIERI, M. et. al (2012).

Para a testemunha (sem tratamento), à medida que aumenta o tempo (horas) diminui tanto o comprimento de parte aérea como a parte radicular, conforme as Figuras 4 e 5, respectivamente. Ao considerar os fatores comprimento de parte aérea e de parte radicular, no tratamento com Vincit ${ }^{\circledR} 50 S C$, nota-se que há um decréscimo considerável no tempo zero em relação aos demais tempos. Isso ocorre devido às sementes estarem muito tempo em contato com temperaturas elevadas, fazendo com que as mesmas não se desenvolvam uniformemente. Conforme Ramos et al. (2004), constataram que o estresse provocado pelo teste de envelhecimento acelerado tradicional a $45^{\circ} \mathrm{C}$ por 48 horas foi suficiente para ocasionar a morte de sementes de rúcula.

Deste modo, o tratamento químico não mostrou diferença significativa quando comparado aos demais tratamentos. Contudo, quando utilizado o produto químico associado com o biológico (Vincit ${ }^{\circledR} 50 \mathrm{SC}+$ Trichodel $^{\circledR}$ ), as variáveis parte aérea e de parte radicular não houve diferença para a Testemunha (sem tratamento) e o tratamento somente com Vincit ${ }^{\circledR} 50 \mathrm{SC}$ (Figuras 4 e 5 , respectivamente). Portanto, as sementes tratadas com o produto Trichodel ${ }^{\circledR}$ apresentaram um comportamento distinto dos demais, porém, com uma queda numérica menos drástica.

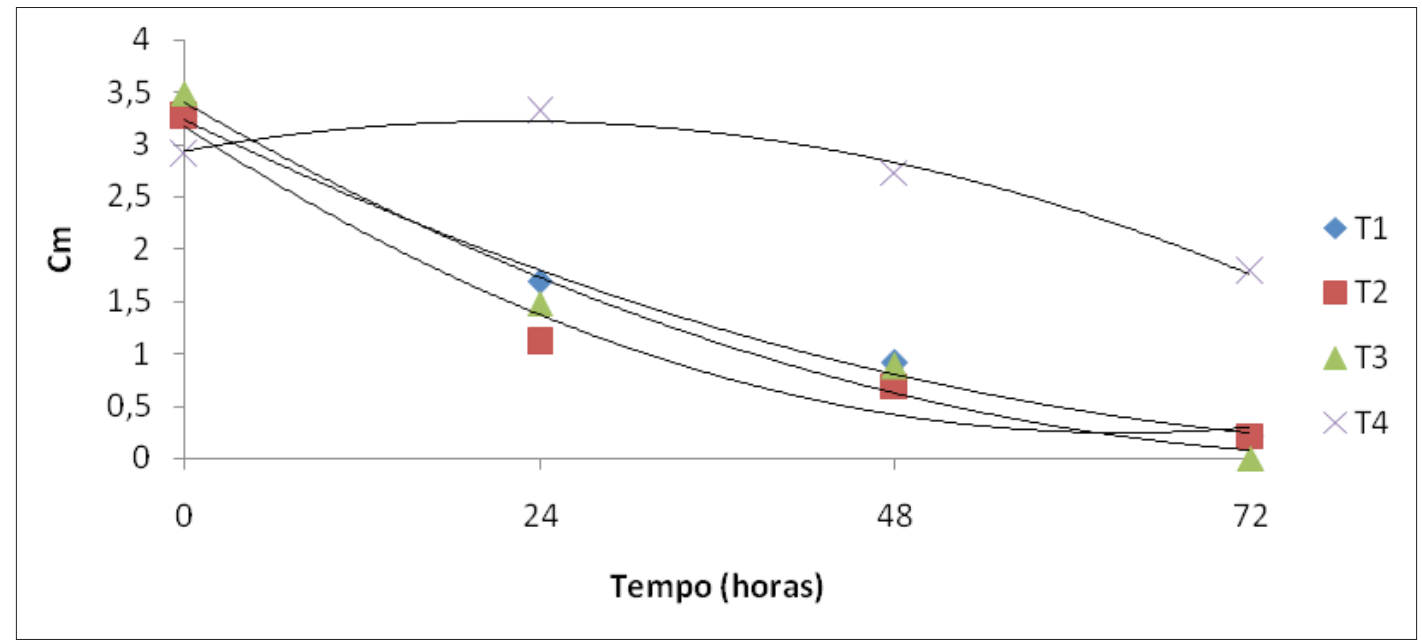

Figura 4 - Comprimento de parte aérea $(\mathrm{cm})$ de sementes de aveia preta cv. Comum após diferentes tempos de envelhecimento acelerado. T1 - Testemunha; T2 - Tratamento químico (Vincit $\left.{ }^{\circledR} 50 S C\right)$; T3 - Químico + Trichoderma $\left(\right.$ Vincit $^{\circledR} 50 \mathrm{SC}+$ Trichodel $^{\circledR}$ ) e T4 - Trichoderma (Trichodel ${ }^{\circledR}$ ). Santa Maria, 2012.

Fonte: Dados da pesquisa. BARBIERI, M. et. al (2012). 
Tabela 4 - Equações das análises de regressão da variável comprimento de parte aérea $(\mathrm{cm})$ de Avena strigosa cv. comum submetidas ao envelhecimento acelerado sob diferentes tratamentos.

\begin{tabular}{|c|c|c|}
\hline \multicolumn{3}{|c|}{ Comprimento de parte aérea $(\mathrm{cm})$} \\
\hline Tratamento & Equação das análises de regressão & Nível de significância \\
\hline Testemunha - T1 & $\mathrm{y}=0,000 \mathrm{x}^{2}-0,069 \mathrm{x}+3,235$ & $\mathrm{R}^{2}=0,995$ \\
\hline Vincit $^{\circledR} 50 \mathrm{SC}-\mathrm{T} 2$ & $\mathrm{y}=0,000 \mathrm{x}^{2}-0,092 \mathrm{x}+3,176$ & $\mathrm{R}^{2}=0,969$ \\
\hline Vincit $^{\circledR} 50 \mathrm{SC}+$ Trichodel $^{\circledR}-\mathrm{T} 3$ & $\mathrm{y}=0,000 \mathrm{x}^{2}-0,081 \mathrm{x}+3,404$ & $\mathrm{R}^{2}=0,978$ \\
\hline Trichodel $^{\circledR}-\mathrm{T} 4$ & $\mathrm{y}=-0,000 \mathrm{x}^{2}+0,026 \mathrm{x}+2,931$ & $\mathrm{R}^{2}=0,984$ \\
\hline
\end{tabular}

Fonte: Dados da pesquisa. BARBIERI, M. et. al (2012).

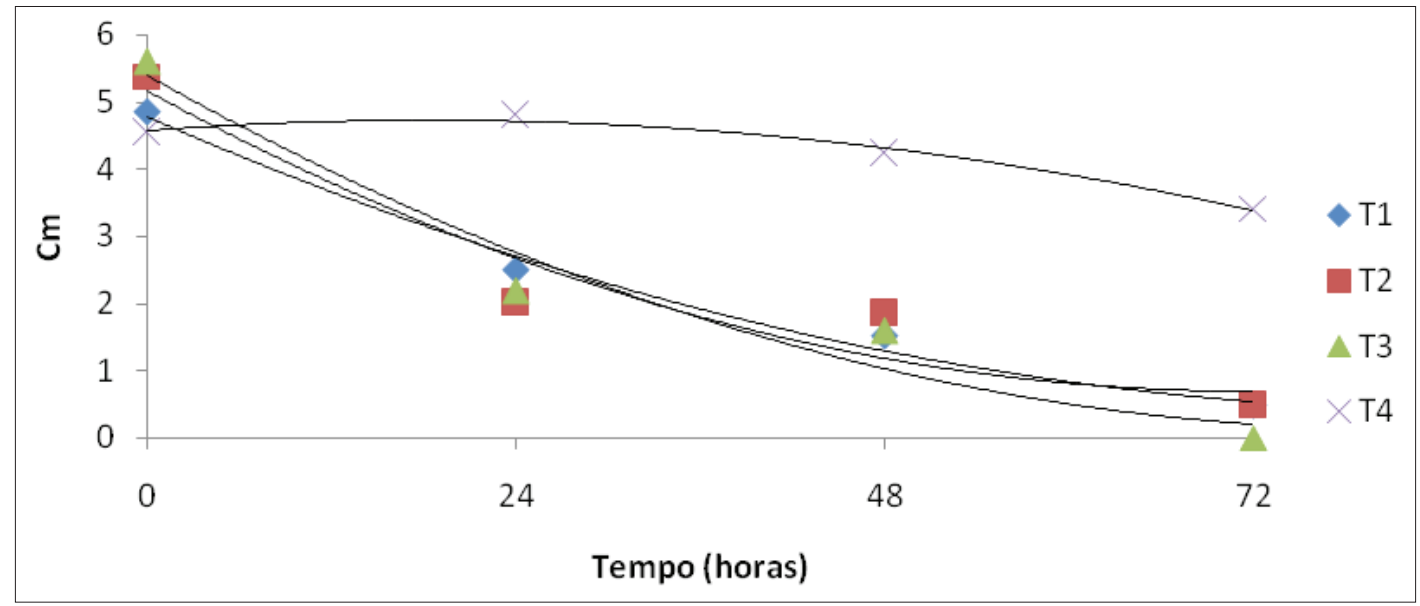

Figura 5 - Comprimento de raiz $(\mathrm{cm})$ de sementes de aveia preta cv. Comum após diferentes tempos de envelhecimento acelerado. T1 Testemunha; T2 - Tratamento químico (Vincit $\left.{ }^{\circledR} 50 S C\right)$; T3 - Químico + Trichoderma (Vincit ${ }^{\circledR} 50 \mathrm{SC}+$ Trichodel $^{\circledR}$ ) e T4 - Trichoderma (Trichodel $^{\circledR}$ ). Santa Maria, 2012.

Fonte: Dados da pesquisa. BARBIERI, M. et. al (2012).

Tabela 5 - Equações das análises de regressão da variável comprimento de (\%) de Avena strigosa cv. comum submetidas ao envelhecimento acelerado sob diferentes tratamentos.

\begin{tabular}{|c|c|c|}
\hline \multicolumn{2}{|c|}{ Comprimento de raiz $(\mathrm{cm})$} \\
\hline Tratamento & Equação das análises de regressão & Nível de significância \\
\hline Testemunha - T1 & $\mathrm{y}=0,000 \mathrm{x}^{2}-0,099 \mathrm{x}+4,772$ & $\mathrm{R}^{2}=0,990$ \\
\hline Vincit $^{\circledR} 50 \mathrm{SC}-\mathrm{T} 2$ & $\mathrm{y}=0,000 \mathrm{x}^{2}-0,124 \mathrm{x}+5,158$ & $\mathrm{R}^{2}=0,924$ \\
\hline Vincit $^{\circledR} 50 \mathrm{SC}+$ Trichodel $^{\circledR}-\mathrm{T} 3$ & $\mathrm{y}=0,000 \mathrm{x}^{2}-0,129 \mathrm{x}+5,404$ & $\mathrm{R}^{2}=0,956$ \\
\hline Trichodel $^{\circledR}-\mathrm{T} 4$ & $\mathrm{y}=-0,000 \mathrm{x}^{2}+0,016 \mathrm{x}+4,567$ & $\mathrm{R}^{2}=0,983$ \\
\hline
\end{tabular}

Fonte: Dados da pesquisa. BARBIERI, M. et. al (2012).

\section{CONCLUSÕES}

O tratamento biológico foi bastante promissor, principalmente para o teste de vigor e germinação. Isso demonstrou que nem mesmo o produto químico do grupo dos triazóis inibiu a colonização do fungo na semente, pois a pre- sença do gênero Trichoderma spp. foi constante. O tratamento com o produto biológico Trichodel ${ }^{\circledR}$, foi promissor para a germinação, crescimento de plântulas e raiz, e demonstrou decréscimo das plântulas anormais e sementes mortas. 


\section{REFERÊNCIAS}

BARNETT, H. L.; HUNTER, B. B. Illustred genera of imperfect fungi.3Ed. Minnesota: Burgess Publishing Company, 1999, p. 241.

BALARDIN, R.S.; LOCH, L.C. Efeito de thiram sobre a germinação de sementes de centeio e aveia. Revista Brasileira de Sementes, v.9, n.1, p.113-117, 1987.

BARBOSA, M.A.G.; REHN; K.G.; MENEZES, M.; MARIANO, R.L.R. Antagonismof Trichoderma speciesonCladosporumherbarumandtheirenzymaticcharacterization. Brazilian Journal of Microbiology, v.32, p.98-104, 2001.

BASRA, A. S. Seed quality - Basic mechanisms and agricultural implications. FoodsProducts Press, 1994. $389 \mathrm{p}$.

BRASIL, Ministério da Agricultura e Reforma Agrária. Regras para análises de sementes. Brasília, DF: SNAD/CLAV, 1992. 365p.

BEVILAQUA, G.A.; PIEROBOM, C.R. Qualidade sanitária e fisiológica de sementes de aveia-preta (Avena strigosa Schreb) da zona sul do Rio Grande do Sul. Revista Brasileira de Sementes, v.17, n.1, p.19-22, 1995.

CARVALHO, N. M. Vigor de sementes. In: CÍCERO, S. M.; MARCOS FILHO, J. \& SILVA, W. R. (Coord.). Atualização em produção de sementes. Campinas: Fundação Cargill, 1986. p. 207-223.

\section{COMISSÃO BRASILEIRA DE PESQUISA DE} AVEIA. In: Indicações técnicas para cultura aveia. Guarapuava: A Comissão: Fundação Agrária de Pesquisa Agropecuária, 2006.

CORREAA, E.B.; BETTIOL, W. Controle Biológico da Podridão de Raízes Causada por Pythium spp. em Cultivos Hidropônicos. Jaguariúna: Embrapa Meio Ambiente, 2009, pg. 26 - (Embrapa Meio Ambiente. Documentos; 77).

DIAS, M.C.L.L. \& BARROS, A.S.R. Avaliação da qualidade de sementes de milho. Londrina: IAPAR, 1995. 43p. (Circular, 88).

ETHUR, L.Z.; MENEZES, J.P.; CAMARGO, R.F. et al. Diferentes produtos na sanidade e vigor de sementes de zínia (Zinniaelegans).In: III CON-
GRESSO BRASILEIRO DE AGROECOLOGIA, 2005. Florianópolis. Anais III Congresso Brasileiro de Agroecologia. Florianópolis, 2005.

ETHUR, L.Z.; ROCHA, E.K. da; MILANESI, P.; MUNIZ,M.F.B.; BLUME. E. Sanidade de sementes e emergência de plântulas de nabo forrageiro, aveia preta e centeio submetidas a tratamentos com bioprotetor e fungicida. Ciência e Natura, UFSM, v. 28, n. 2, p. $17-27,2006$.

FARIA, A.Y.K.; ALBUQUERQUE, M.C.F.; NETO, D.C. Qualidade fisiológica de sementes de algodoeiro submetidas a tratamentos químico e biológico. Revista Brasileira de Sementes, v.25, n.1, p.121-127, 2003.

FARIAS, C.R.J. et al., Qualidade sanitária de sementes de aveia-preta (avena strigosa schreb.) produzidas no estado do rio grande do sul, safra 1999/2000. Revista Brasileira de Sementes, v. 24, n 1, p.1-4, 2002.

FARIAS, C.R.J.; DEL PONTE, E.M.; LUCCA FILHO, O.A.; PIEROBOM, C.R. Fungos causadores de helmintosporiose associados às sementes de aveia -preta (Avena strigosa, schreb.). Revista Brasileira Agrociência, Pelotas, v.11, n.1, p.57-61, 2005.

FERREIRA, D.F. SISVAR: um programa para análises estatísticas e ensino de estatística. Revista Symposium, v.6, p.36-41, 2008.

FORCELINI, C.A. Tratamento de sementes de trigo no Brasil. In: MENTEN, J.O.M. Patógenosem sementes: deteç̧ão, danos e controle químico. São Paulo: Ciba Agro, 1995. p. 247 - 264.

HARMAN, G.E. Myth and dogmas of biocontrol changes in perceptions derived from research on Trichoderma harzianum T-22. PlantDisease, v. 84, n.4, p. 377-393, 2000.

HENNING, F.A.; MERTZ, L.M.; ZIMMER, P.D.; TEPLIZKY, M.D.F. Qualidade fisiológica, sanitária e análise de isoenzimas de sementes de aveia-preta tratadas com diferentes fungicidas. Revista Brasileira de Sementes, v. 31, n. 3, p.063-069, 2009.

HENNING, A.A.; HENNING, F.A. Microbiolization of soybean seeds.In: XIX Seminário Pan Americano de Semillas, Assunção. Conferencias y Resumenes de Trabajos Presentados. Assunción: FELAS, 2004. p. 330. 
HENNING, F. A. et al. Qualidade fisiológica, sanitária e análise de isoenzimas de sementes de aveia-preta tratadas com diferentes fungicidas.

Revista Brasileira de Sementes, vol. 31, n 3 , p.063069, 2009.

LIMA e BORGES, E. E. de\& RENA, A. B. Germinação de sementes. In: Sementes florestais tropicais. Brasília: ABRATES, 1993.

MACHADO, J.C.; WAQUIL, J.M.; SANTOS, J.P.; REICHENBACH, J.W.; Tratamento de sementes no controle de fitopatógenos e pragas. Informe Agropecuário, Belo Horizonte, v.27, n.232, p.76-87, maio/ jun. 2006.

MACIEL, C.G,; SANTOS, R.F.; MEZZOMO, R.; WEBER, M.N.D.; MUNIZ, M.F.B.; BLUME, E.; Germinação e Sanidade de Sementes de Ipê-amarelo (Tabebuia serratifolia), após Submissão a Diferentes Tratamentos. Revista Brasileira de Agroecologia, v.4, n.2, p. $952-955,2009$.

MARTINS-CORDER, M.P.P.; MELO, I.S. Influência de Trichoderma viridee $T$. koningiina emergência de plântulas e no vigor de mudas de berinjela. Revista Brasileira de Biologia, v.57, n.1, p.39-45, 1997.

MATTHEWS, S. Physiology of seed ageing. Outlook on Agriculture, Elmsford, v.14, n.2, p.8994, 1985.

MENEZES, N.L. et al. Teste de condutividade elétrica em sementes de aveia preta. Revista Brasileira de Sementes, vol. 29, n 2, p.138-142, 2007.

MARCOS FILHO, J. Teste de envelhecimento acelerado. In: KRZYZANOWSKI, F.C.; VIEIRA, R.D.; FRANÇA NETO, J.B. (Ed.) Vigor de sementes: conceitos e testes. Londrina: Abrates, 1999. p.3.13.24 .

MARCOS FILHO, J.; CÍCERO, S.M.; SILVA, W.R. Avaliação da qualidade das sementes. Piracicaba: FEALQ, 1987. 230p.

McDONALD, M.B.; GUPTA, I.J.; SCHMITTHENNER, A.F. Effect of storage fungi on seed vigour of soybean. Seed Science and Technology, Zurich, v.21, n.3, p.581-591, 1993.

McDONALD Jr., M.B. Assessment for seed quality. HortScience, v.15, n.6, p.784- 788, 1980.
MELLO, V.D.C.; TILLMANN, M.A.A. O teste de vigor em câmara de envelhecimento

precoce. Revista Brasileira de Sementes, Brasília, v. 9, n. 2, p. 93-102, 1987.

MEHTA, Y.R. Ocorrência de Drechsleraspp. em aveia branca no Estado do Paraná. Summa Phytopathologica, São Paulo, v. 25, n. 3, p. 265-267, 1999.

NEERGAARD, P. Seed Pathology. 2. ed. London, MacMillan Press, v.2, 1979.

PANOBIANCO, M.; MARCOS FILHO, J. Comparação entre métodos para avaliação da qualidade fisiológica de sementes de pimentão. Revista Brasileira de Sementes, v.20, p.306-310, 1998.

RAMOS, N.P.; FLOR, E.P.O.; MENDONÇA, E.A.F.; MINAMI, K. Envelhecimento acelerado em sementes de rúcula (Eruca sativa L.). Revista Brasileira de Sementes, Brasília, v.26, n.1, p.98-103, 2004.

SCHUCH, L.O.B. e LIN, S.S. Efeito do envelhecimento rápido sobre o desempenho de sementes e de plantas de trigo. Pesquisa Agropecuária Brasileira, Brasília, v.17, n.8, p.1163-1170, 1982.

SCHUCH, L.O.B. Vigor das sementes e aspectos da fisiologia da produção em aveia-preta (Avena strigosa Schreb.). Pelotas:Universidade Federal de Pelotas, 1999. 127p. (Tese Doutorado).

SCHUCH, L.O.B.; NEDEL, J.L.; ASSIS, F.N. \& MAIA, M.S. Crescimento em laboratório de plântulas de aveia-preta (Avenastrigosa Schreb.) em função do vigor das sementes. Revista Brasileira de Sementes, Brasília, v.21, n.1, p.229-234, 1999.

SOAVE, J.; WETZEL, M.M.V. Patologia de sementes. Campinas: Fundação Cargill, 1987. 480p.

SOUZA, S.A.; NAKAGAWA, J.; MACHADO, C.G.Teste de envelhecimento acelerado em sementes de aveia preta. Revista Brasileira de Sementes, v.31, n.2, p.155-163, 2009.

TORRES, S.B. Métodos para avaliação do potencial fisiológico de sementes de melão.2002. 103f. Tese (Doutorado em Agronomia) - ESALQ/USP, Piracicaba. 